BULL. AUSTRAL. MATH. SOC.

$90 C 30,90 C 20$

VOL. 9 (1973), 249-257.

\title{
On the complex complementarity problem
}

\section{Bertram Mond}

The complex linear complementarity problem considered here is the following: Find $z$ such that

$$
\begin{gathered}
M z+q \in S^{*}, \quad z \in S, \\
\operatorname{Re}\left[z^{H} M z+z^{H} q\right]=0,
\end{gathered}
$$

where $S$ is a polyhedral convex cone in $c^{p}, S^{*}$ the polar cone, $M \in C^{p^{\times p}}$ and $q \in C^{p}$.

Generalizing earlier results in real and complex space, it is shown that if $M$ satisfies $\operatorname{Re} z^{H} M z \geq 0$ for all $z \in C^{D}$ and if the set satisfying $\mathrm{Mz}+q \in S^{*}, z \in S$ is not empty, then a solution to the complex linear complementarity problem exists. If $\operatorname{Re}^{H} M z>0$ unless $z=0$, then a solution to this problem always exists.

\section{Introduction}

Recently, McCallum [8] introduced the complex linear complementarity problem and established conditions for the existence of solutions. McCallum dealt with the problem where each of the constraints was restricted to a sector in the complex plane. Here we give conditions for the existence of solutions to the more general complex linear complementarity problem where the constraints are restricted to polyhedral cones.

Received 3 May 1973. 


\section{Notation and statement of problem}

For brevity, we shall use much of the notation of [8].

Superscript $H$ denotes conjugate transpose. Let $S$ denote a polyhedral cone in $c^{n}$, then the polar of $S$ is defined by

$$
S^{*}=\left\{w \in C^{n}: z \in S \Rightarrow \operatorname{Re}\left(x^{H} z\right) \geq 0\right\} \text {. }
$$

The following observations follow readily from the definition of $S^{*}$ [2]:

if $S$ and $T$ are polyhedral cones in $C^{n}$, then

$$
(S \times T)^{*}=S^{*} \times T^{*} ;
$$

if $s=c^{n}$, then $S^{*}=\{0\}$;

if $S=R_{+}$, then $S^{*}=\{z \in C: \operatorname{Re} z \geq 0\}$.

The complex complementarity problem (CCP) we shall deal with is the following:

Let $M \in c^{p \times p}, q \in c^{p}, S \subset c^{p}$, a convex polyhedral cone. PROBLEM (CCP). Find $z \in C^{P}$ such that

$$
\begin{gathered}
M z+q \in S^{*}, \\
z \in S, \\
\operatorname{Re}\left[z^{H}(M z+q)\right]=0 .
\end{gathered}
$$

Closely related to this problem is the complex quadratic programing problem.

$$
\begin{aligned}
\text { PROBLEM Q1. Minimize } & f(z) \equiv \operatorname{Re}\left[z^{H} M z+z^{H} q\right] \\
& \text { subject to }(1) \text { and (2). }
\end{aligned}
$$

A solution to Problem (CCP) is clearly an optimal solution of Problem Ql. The reverse need not be true since, for optimal $z_{0}$, we may have $f\left(z_{0}\right)>0$. We shall later state some restrictions on $M$ that assure that $f\left(z_{0}\right)=0$. 


\section{Complex alternative theorems}

Let $A \in c^{m \times n}, A_{1} \in C^{m \times n_{1}}, A_{2} \in C^{m \times n_{2}}$; and let $s \subset c^{n}$, $S_{1} \subset C^{n_{1}}, s_{2} \subset C^{n_{2}}, T \subset C^{m}$ be polyhedral convex cones. We shall make use of the following result of Ben-Israel [3]:

THEOREM 1. The system

$$
A_{1} x_{1}+A_{2} x_{2} \in-T, \quad 0 \neq x_{1} \in S_{1}, \quad x_{2} \in S_{2}
$$

has a solution if, and only if,

$$
A_{1}^{H} y \in \operatorname{int} S_{1}^{*}, \quad A_{2} y \in S_{2}^{*}, \quad y \in T^{*}
$$

has none.

THEOREM 2. The system

$$
A z-b \in T, \quad z \in S
$$

has a solution, if and only if,

$$
-A^{H} v \in S^{*}, \quad v \in T^{*}, \quad \operatorname{Re} b^{H} v>0
$$

has none.

Proof. (6) is equivalent to

$$
-A z+b \xi \in-T, \quad z \in S, \quad 0 \neq \xi \in R_{+} .
$$

By Theorem 1, (8) has a solution, if and only if,

$$
-A^{H} v \in S^{*}, \quad b^{H} v \in \operatorname{int}\left(R_{+}\right)^{*}, \quad v \in T^{*} .
$$

Since $b^{H} v \in \operatorname{int}\left(R_{+}\right)^{*}$ means $\operatorname{Re} b^{H} v>0$, (9) yields (7).

COROLLARY. Let $M \in c^{p \times p}, q \in c^{p}, s \in c^{p}$, a polyhedral convex cone. Then the set satisfying $\mathrm{Mz}+q \in S^{*}, z \in S$ is nonempty if, and only if,

$$
-M^{H} v \in S^{*}, \quad v \in S, \quad \operatorname{Re} q^{H} v<0
$$

has no solution.

Proof. In Theorem 2, take $n=m=p, A=M, b=-q$, and 
$T=S^{*}$.

\section{Complex quadratic programming}

We shall utilize the following duality theorem for complex quadratic programming of $[1]$ :

THEOREM 3. Let $B \in C^{n \times n}$ be positive semi-definite Hermitian. Let $A \in C^{m \times n}, b \in C^{m}, c \in C^{n}$ and $s \subset c^{n}, T \subset C^{m}$ be convex polyhedral cones. Consider the pair of problems

PROBLEM P1. Minimize $F(x) \equiv \operatorname{Re}\left[\frac{3}{3} x^{H} B x+c^{H} x\right]$

$$
\text { subject to } \begin{aligned}
A x-b & \in T, \\
x & \in S .
\end{aligned}
$$

PROBLEM P2. Maximize $g(y, z) \equiv \operatorname{Re}\left[-\frac{1}{2} y^{H} B y+b^{H} z\right]$

$$
\text { s ubject to } \begin{array}{r}
-A^{H} z+B y+c \in S^{*}, \\
z \in T^{*} .
\end{array}
$$

If Problem PI has an optimal solution $x_{0}$, then there exists a vector $z_{0}$ such that $\left(x_{0}, z_{0}\right)$ is an optimal solution of Problem P2; and

$$
F\left(x_{0}\right)=g\left(x_{0}, z_{0}\right)
$$

We want to modify Theorem 3 so as to eliminate the restriction in $\mathrm{Pl}$ that $B$ must be hermitian. We note that, even if $B$ is not hermitian,

$$
B=\left(B+B^{H}\right) / 2+\left(B-B^{H}\right) / 2 \text {. }
$$

Since $z^{H}\left[\left(B-B^{H}\right) / 2\right] z$ is pure imaginary,

$$
\operatorname{Re} z^{H} B z=\operatorname{Re} z^{H}\left[\left(B+B^{H}\right) / 2\right] z,
$$

where $\left(B+B^{H}\right) / 2$ is always hermitian. This suggests the following definitions :

$B \in C^{n \times n}$ (not necessarily hermitian) is said to be positive semi-definite if 


$$
\operatorname{Re} z^{H} B z \geq 0 \text { for all } z \in C^{n}
$$

and positive definite if

$$
\operatorname{Re} z^{H} B z>0 \text { unless } z=0 .
$$

(11) and Theorem 3 lead to the following:

THEOREM 4. If Problem Pl, with $B$ positive semi-definite but not necessarily hermitian, has on optimal solution $x_{0}$, then there exists a vector $z_{0}$ such that $\left(x_{0}, z_{0}\right)$ is an optimal solution of

$$
\begin{array}{r}
\text { PROBLEM P2'. Maximize } g(y, z) \equiv \operatorname{Re}\left[-\frac{3}{2} y^{H} B y+b^{H} z\right] \\
\text { subject to } A^{H} z+\left[\left(B+B^{H}\right) / 2\right] y+c \in S^{*}, \\
z \in T^{*},
\end{array}
$$

and $F\left(x_{0}\right)=g\left(x_{0}, z_{0}\right)$.

Solvability of the complex complementarity problem

We now state some conditions that assure the existence of a solution of Problem (CCP).

THEOREM 5. Let $M \in C^{P^{x p}}$ be positive semi-definite. If the set of feasible solutions of Problem QI is non-empty, then there exists a vector $z_{0}$ such that $f\left(z_{0}\right)=0$.

Proof. The dual of Problem Ql is

PROBLEM Q2. Maximize $G(y, v) \equiv \operatorname{Re}\left[-y^{H} M y-v^{H} q\right]$

$$
\text { subject to } \begin{array}{r}
M^{H} v+M y+M^{H} y+q \in S^{*}, \\
v \in S .
\end{array}
$$

Since the feasible set of $Q 1$ is nonempty and the quadratic objective function is bounded below (by zero), Ql achieves a minimum for some optimal vector $z_{0}[6],[8]$. By Theorem 4 , there exists $v_{0}$ such that $\left(z_{0}, v_{0}\right)$ is optimal for $Q 2$ and 


$$
f\left(z_{0}\right)=\operatorname{Re}\left[z_{0}^{H} M z_{0}+z_{0}^{H} q\right]=\operatorname{Re}\left[-z_{0}^{H} M z_{0}-v_{0}^{H} q\right]=G\left(z_{0}, v_{0}\right) .
$$

From (15) and (1),

$$
\operatorname{Re}\left[v_{0}^{H} M z_{0}+v_{0}^{H} q\right] \geq 0
$$

From (2) and (14),

$$
\operatorname{Re}\left[-z_{0}^{H} M^{H} v_{0}+z_{0}^{H} M z_{0}+z_{0}^{H} M^{H} z_{0}+z_{0}^{H} q\right] \geq 0
$$

Noting that $\operatorname{Rev} v_{0}^{H} z_{0}=\operatorname{Re} z_{0}^{H}{ }^{H} v_{0}$, and that $\operatorname{Re} z_{0}^{H} M z_{0}=\operatorname{Re} z_{0}^{H}{ }^{H} z_{0}$, adding (17) and (18) gives

$$
\operatorname{Re}\left[-v_{0}^{H} q-z_{0}^{H} M z_{0}\right] \leq \operatorname{Re}\left[z_{0}^{H} M z_{0}+z_{0}^{H} q\right] .
$$

From (16), the inequality in (19), and hence also in (17) and (18) must be satisfied as equalities, that is,

$$
\begin{gathered}
\operatorname{Re}\left[v_{0}^{H} M z_{0}+v_{0}^{H}{ }^{H}\right]=0, \\
\operatorname{Re}\left[-z_{0}^{H} M^{H} v_{0}+z_{0}^{H} M z_{0}+z_{0}^{H} M^{H} z_{0}+z_{0}^{H} q=0 .\right.
\end{gathered}
$$

(1) and (2) give

$$
\operatorname{Re}\left[z_{0}^{H} M z_{0}+z_{0}^{H} q\right] \geq 0
$$

(14) and (15) give

$$
\operatorname{Re}\left[-v_{0}^{H} M^{H} v_{0}+v_{0}^{H} M z_{0}+v_{0}^{H} M^{H} z_{0}+v_{0}^{H} q\right] \geq 0 .
$$

(20) and (23) yield

$$
\operatorname{Re}\left[-v_{0}^{H} M^{H} v_{0}+v_{0}^{H}{ }^{H} z_{0}\right] \geq 0
$$

From (21) and (22),

$$
\operatorname{Re}\left[z_{0}^{H} M z_{0}+z_{0}^{H} q\right]=\operatorname{Re}\left[z_{0}^{H} M^{H} v_{0}-z_{0}^{H} M^{H} z_{0}\right] \geq 0 .
$$

Adding (24) and (25) and noting the positive semi-definiteness of $M$, we obtain 
(26) $0 \leq \operatorname{Re}\left[z_{0}^{H} M z_{0}+z_{0}^{H} q\right] \leq \operatorname{Re}\left[-z_{0}^{H}{ }^{H} z_{0}-v_{0}^{H} M^{H} v_{0}+v_{0}^{H}{ }^{H} z_{0}+z_{0}^{H} M^{H} v_{0}\right]$

$$
=-\operatorname{Re}\left[\left(z_{0}-v_{0}\right)^{H} M^{H}\left(z_{0}-v_{0}\right)\right]=-\operatorname{Re}\left[\left(z_{0}-v_{0}\right)^{H} M\left(z_{0}-v_{0}\right)\right] \leq 0 .
$$

Thus equality holds throughout (26) giving

$$
f\left(z_{0}\right)=\operatorname{Re}\left[z_{0}^{H} M z_{0}+z_{0}^{H} q\right]=0
$$

THEOREM 6. Let $M \in c^{p \times p}$ be positive definite. Then the set of feasible solutions of Problem $Q 1$ is non-empty and there exists an optimal vector $z_{0}$ such that (27) holds.

Proof. Suppose that the feasible set of Ql is empty. By the Corollary to Theorem 2, the system

$$
\begin{gathered}
-M^{H} v \in S^{*}, \quad v \in S, \\
\operatorname{Req}^{H} v<0
\end{gathered}
$$

has a solution. (28) implies

$$
\operatorname{Re}-v^{H} M^{H} v=\operatorname{Re}-v^{H} M v \geq 0 .
$$

Since $M$ is positive definite, $v$ must be the zero vector, contradicting (29). Hence the feasible set is nonempty.

The remainder of Theorem 6 now follows from Theorem 5 .

\section{Special cases}

If

$$
S=\left\{z \in C^{n}:|\arg z| \leq \beta\right\}
$$

and

$$
T=\left\{w \in C^{m}:|a r g w| \leq \alpha\right\}
$$

for given $\beta \in R_{+}^{n}, \alpha \in R_{+}^{m}, \beta_{i} \leq \pi / 2, i=1, \ldots, n ; \alpha_{i} \leq \pi / 2$, $i=1, \ldots, m$, then Theorem 2 yields a result of Mond and Hanson ([10], Theorem 8). If all vectors and matrices are real and $S=R_{+}^{n}, T=R_{+}^{m}$, then Theorem 2 yields an alternative theorem in real space of Gale ([7], 
p. 47).

If $S$ is defined by (30) with $n=p$, Theorem 5 gives the corresponding result of MCCallum [8]. If $S=R_{+}^{n}$ and all matrices and vectors are real, then Theorem 5 reduces to the result of Cottle [4] while Theorem 6 gives the corresponding existence theorem of Dorn [5].

\section{References}

[1] Robert A. Abrams and Adi Ben-Israel, "A duality theorem for complex quadratic programming", J. Optimization Theory App Z. 4 (1969), 244-252.

[2] Adl Ben-Israel, "Linear equations and inequalities on finite dimensional, real or complex, vector spaces: a unified theory". J. Math. Anal. Appl. 27 (1969), 367-389.

[3] A. Ben-Israel, "Theorems of the alternative for complex linear inequalities", Israel J. Math. 7 (1969), 129-136.

[4] Richard W. Cottle, "Note on a fundamental theorem in quadratic programming", J. Soc. Indust. App 2. Math. 12 (1964), 663-665.

[5] W.S. Dorn, "Self-dual quadratic programs", J. Soc. Indust. Appl. Math. 9 (1961), 51-54.

[6] Marguerite Frank and Philip Wolfe, "An algorithm for quadratic programming", Naval Res. Logist. Quart. 3 (1956), 95-110.

[7] David Gale, The theory of linear economic models (McGraw-Hill, New York, Toronto, London, 1960).

[8] Charles J. McCallum, Jr, "Existence theory for the complex linear complementarity problem", J. Math. Anal. Appl. 40 (1972), $738-762$.

[9] Bertram Mond and Morgan A. Hanson, "Symmetric duality for quadratic programming in complex space", J. Math. Anal. Appl. 23 (1968), 284-293. 
[10] Bertram Mond and Morgan A. Hanson, "Some generalizetions and applications of a complex transposition theorem", Linear Algebra and App Z. 2 (1969), 401-411.

Department of Mathematics,

La Trobe University,

Bundoora,

victoria. 